\title{
Comparative efficacy of herbal methionine vis-a-vis dl-methionine on performance of broiler chicken
}

\author{
D. Bhutyal, N. Khan", R.K. Sharma, V. Mahajan and J.S. Sasan
}

Division of Instructional Livestock Farm Complex, Faculty of Veterinary Sciences \& Animal Husbandry, Sher-E-Kashmir University of Agricultural Sciences \& Technology of Jammu, R.S. Pura

*Corresponding Author: nazamdrkhan@gmail.com

Journal of Livestock Science (ISSN online 2277-6214) 13:48-57

Received on 5/11/21; Accepted on 1/1/22; Published on 10/1/22

doi. 10.33259/JLivestSci.2022.48-57

\begin{abstract}
The present study was designed with the objective to evaluate the effect of replacement of DLmethionine (DL-M) with graded levels of Herbal methionine (HM) on performance of broiler chicken. For the study, two hundred forty day old unsexed broiler chicks were procured, weighed and randomly divided into five dietary treatments $\left(\mathrm{C}, \mathrm{T}_{1}, \mathrm{~T}_{2}, \mathrm{~T}_{3}\right.$ and $\mathrm{T}_{4}$ having $0,25,50,75$ and $100 \%$ replacement of DL-M with HM, respectively; $n=48 /$ group). There were twelve birds in each replicate. The diets formulated were iso-caloric and iso-nitrogenous. The results revealed significantly $(\mathrm{P}<0.05)$ higher body weight gain $(\mathrm{g})$ in $\mathrm{C}$ than $\mathrm{T}_{1}$. The cumulative FCR of whole trial $(0-6$ week) was significantly higher $(\mathrm{P}$ $<0.05)$ in $\mathrm{T}_{4}(2.20)$ than $\mathrm{T}_{1}(2.03), \mathrm{T}_{2}$ (2.07) and $\mathrm{T}_{3}$ (2.10), but was significantly better in $\mathrm{C}$ (1.91). PER after six week was higher in $\mathrm{C}$ followed by $\mathrm{T}_{1}$ and least in $\mathrm{T}_{4}$ with variable results for rest of dietary treatments. Different carcass characteristics and cut up parts were found higher in DL - M fed birds than HM fed group, but spleen weight was found higher in HM fed group. No effect of HM supplementation was seen on cost economics. Feather quality was found better in HM fed groups. It was concluded that dietary replacement of DL methionine with variable levels of herbal methionine does not seem to impart any beneficial effect on performance of broiler chicken except feather quality of birds.
\end{abstract}

Keywords: broilers; DL-methionine; herbal methionine; feather quality 


\section{Introduction}

Amino acid has noteworthy importance in poultry nutrition and their adequate dietary levels are vital for optimum growth performance of commercial broilers (Ojano-Dirain and Waldroup 2002). Broilers are mostly fed maize-soybean meal based diets in which methionine is the most limiting amino acid (Bhutyal et al. 2019). Methionine is important for energy production, protein synthesis and is essential for several body reactions (Binder 2003). It is essential for synthesis of epinephrine, choline, carnithine and creatine, as it is an important methyl donor. Its supplementation alters immune responses and is beneficial for reducing immunologic stress (Kaur et al. 2013). It protects young chicks from neurological symptoms (Fikre et al. 2010) and also reduces the risk of necrotic enteritis (Dahiya et al. 2007). It is metabolically linked with cystine and choline and is necessary for producing keratins used in feather growth (NRC 1994).

Although, protein in animal sources has adequate methionine levels, but it is not practical to fulfill the methionine requirement of bird ration by incorporating animal protein sources (Dafwang 2006). The common source of methionine in poultry diet is DL-methionine (DL-M), which is produced by chemical synthesis from acrolein, methyl mercaptan and hydrogen cyanide (Figge et al. 2010). The fluctuating prices for petrol derived precursors of acrolein and methyl mercaptan is responsible for variable price of DL-M and increases the cost of finished product/finished feed. Also, its excess quantity in the diet is metabolized into highly toxic compounds and its usage is questioned in organic poultry production also.

Methionine is present naturally in plants in dipeptide and oligopeptide forms and also concomitantly enzymes are present in plants for conversion of methionine into L-isomer (active form) for its optimum utilization. Thus, Herbal methionine (HM) may be an effective source and may replace DL$\mathrm{M}$ in poultry ration.. The literature pertaining to use of HM in poultry ration as a replacement of DL-M is scanty, therefore the present study is designed to evaluate the effect of replacement of DL-M with graded levels of HM on performance of broiler chicken.

\section{Materials and methods}

The present study was conducted at poultry farm of SKUAST-J. Two hundred forty, day old unsexed broiler chicks (Cobb strain) were purchased from Cheema Farms Pvt. Ltd. Jammu and randomly distributed into five groups of forty eight birds each. The birds were raised in partitioned deep litter houses. Each group was sub-divided into four replicates of twelve birds each in a complete randomized block design. Birds in control group (C) were fed maize soya based diet supplemented with 100\% DL methionine (DL-M) where as in $\mathrm{T}_{1}, \mathrm{~T}_{2}, \mathrm{~T}_{3}$ and $\mathrm{T}_{4}$ groups DL-M was replaced with 25, 50, 75 and $100 \%$ herbal methionine (HM), respectively (HM- METHIOMAX donated by Alphafacts heatlh solutions, free of cost).

The diets formulated were iso-caloric and iso-nitrogenous as per ICAR (2013). The ingredients and chemical composition of broiler pre-starter, starter and finisher chicks is presented in Table 1. HM used in the present study was the mixture of various herbs including Andrographis paniculta (40\%), Ocimum sanctum (30\%) and Azadirachta indica (30\%). The whole experimental trial lasted for 42 days.

Feed intake in each treatment group was recorded on daily basis and body weight of birds was recorded at weekly intervals. The feed samples were analyzed for proximate analysis as per AOAC (2012). At the end of 6 weeks, two birds from each replicate, making a total of eight birds from each treatment were randomly picked, weighed and slaughtered to study carcass characteristics. The parameters studied were live weight, bled weight, eviscerated weight, weight of giblets (heart, liver and gizzard), immunological organ weight (spleen, thymus and bursa of fabricus), weight of viscera and length of intestines. Feather quality was also evaluated at the end of experimental trial in eight birds per group ( 2 birds per replicate). The feather scoring system was applied to back area, wings and tail of bird as described by Kretzschmar-McCluskey et al. (2014). Also visual observation of feather color was done in these birds. Feather weight (without skin) was also estimated by subtracting defeathered weight (g) from bled weight of birds ( $\mathrm{g}$ ). Defeathered weight was estimated by plucking the feathers after dipping the bird in boiled water followed by light singeing of birds.

The data were analyzed by using one way analysis of variance as per the methods of Snedecor and Cochran (1994). The means in different treatments were subjected to Duncan's multiple range test (DMRT) (1995) for ranking mean values of different parameters. 


\section{Results and discussion}

The data on feed intake, body weight gain, feed conversion ratio (FCR) and protein efficiency ratio (PER) of broiler chicks in different groups have been presented in Table 2. Feed intake (g) during different phases viz. pre-starter ( 0 - 2 week), starter (2 - 3 week) and finisher phase (3 - 6 week) showed non-significant difference among different experimental groups. Also, the total feed intake ( $\mathrm{g}$ ) during the entire experimental trial ( 0 - 6 weeks) remained unaffected by different dietary treatments. This observation is not surprising since the metabolizable energy concentrations of the diets were similar and for the fact that birds eat to satisfy their energy requirements. Similar results were reported by earlier researchers on replacing DL-M with HM either completely or in graded levels in broiler diets (Yuan et al. 2012, Sharma and Ranjan 2015, Makinde et al. 2017). However, these results are contradictory to the findings of Salome et al. (2010) who reported significantly $(\mathrm{P}<0.05)$ higher feed intake in $0.25 \%$ DL-M fed group (1330.63 g) in comparison to $0.25 \%(1200.80 \mathrm{~g})$ and $0.5 \%$ (1133.37 g) HM supplemented groups in broiler chickens during 4 week experimental trial, but no difference $(\mathrm{P}>0.05)$ was found on comparison with $1 \% \mathrm{HM}$ supplemented group (1242.20 g). The difference found may be attributed to different HM source used in the two studies.

However, weight gain $(\mathrm{g})$ of pre-starter phase was statistically higher $(\mathrm{P}<0.05)$ in $\mathrm{C}(357.34)$ than $\mathrm{T}_{4}$ (318.81) with intermediate values for $\mathrm{T}_{1}(346.45), \mathrm{T}_{2}$ (348.44) and $\mathrm{T}_{3}$ (344.00). But, during starter phase weight gain was statistically similar among different dietary treatments. However, in finisher phase (3 - 6 week) weight gain $(\mathrm{g})$ was statistically higher $(\mathrm{P}<0.05)$ in $\mathrm{C}(1324.25)$ than rest of the groups (1203.87, 1163.79, 1181.67 and 1161.78 in $\mathrm{T}_{1}, \mathrm{~T}_{2}, \mathrm{~T}_{3}$ and $\mathrm{T}_{4}$, respectively). Likewise for entire trial $(0-6$ week), body weight gain $(\mathrm{g})$ was found significantly higher $(\mathrm{P}<0.05)$ in $\mathrm{C}(2039.60)$ than $\mathrm{T}_{1}(1919.19)$ and was least in $\mathrm{T}_{4}(1794.77)$, but the values of $\mathrm{T}_{2}(1862.93)$ and $\mathrm{T}_{3}(1878.12)$ were statically similar to $\mathrm{T}_{1}$ and $\mathrm{T}_{4}$. The increase in body weight and weight gain is attributed to the potential role of methionine in promoting growth. It may be inferred that DL-M is a better source of methionine than HM. But among the HM groups, $25 \%$ replacement of DL-M with HM showed better results. These results are in line with the findings of Kaur et al. (2013) who also reported higher body weight in broilers fed diets supplemented with synthetic methionine (SM). Contrary to our findings, some authors reported no effect of replacing DL-M with HM on body weight and body weight gain in broilers (Ahmed and Abbas 2015, Makinde et al. 2017). Whereas several researchers found either higher body weight or body weight gain on replacing DL-M with HM either completely or in graded levels in broilers (Kumari et al. 2012). The differences found may be attributed to use of different herbal methionine source in these studies.

The cumulative FCR of pre-starter, starter, finisher and overall trial was better in synthetic methionine fed group, but it is comparable up to $75 \%$ replacement of DL-M with HM. However periodically, the better FCR in pre-starter and starter phase in comparison to finisher phase clearly indicated that the broiler production was economical and more profitable up to $4^{\text {th }}$ week, and after $4^{\text {th }}$ week onwards, it becomes uneconomical. Similarly, Kumari et al. (2012) stated that FCR in broiler chickens increased on supplementation of HM + DL-M in comparison to DL-M supplemented group. Likewise Salome et al. (2010) reported superior FCR in SM fed group (0.25\%) in comparison to $0.25 \%$, $0.50 \%$ and $0.1 \% \mathrm{HM}$ supplemented groups. The present findings corroborate with earlier reports of Yuan et al. (2012) who also reported better FCR in SM supplemented groups in comparison to HM supplementation. On the other hand, Sharma and Ranjan (2015) reported no difference in FCR on substituting DL-M with HM. Several researchers reported no effect on FCR by replacing SM with HM either completely or partially (Ahmed and Abbas 2015, Makinde et al. 2017). On the contrary, Chattopadhyay et al. (2006) reported superior FCR in broiler chicken on supplementation of $15 \mathrm{~g} \mathrm{HM} / \mathrm{kg}$ diet in comparison to $10 \mathrm{~g} \mathrm{SM} / \mathrm{kg}$ diet and $10 \mathrm{~g} \mathrm{HM} / \mathrm{kg}$ diet supplemented groups. The differences found might be explained in light of fact that higher methionine level promotes growth. Also the differences found might be due to usage of different herbal methionine source.

The cumulative PER of pre-starter and starter phase among all five groups was higher $(\mathrm{P}<0.05)$ in $100 \%$ SM supplemented group than $100 \%$ HM supplemented group with intermediate values for rest of the replacements. Likewise the cumulative PER of finisher phase and entire trial was higher in $\mathrm{C}$ followed by $\mathrm{T}_{1}$ and least in $\mathrm{T}_{4}$ with variable results for rest of dietary treatments. The increased protein efficiency ratio in the SM supplemented group may be attributed to better methionine availability from synthetic source in comparison to herbal source. Among replacements of methionine, $25 \%$ replacement of SM with HM is better than rest of the dietary treatments. Contrary to above results, Ahmed and Abbas (2015) reported that supplementation of broiler diets with $0 \%, 0.15 \% \mathrm{SM}, 0.15 \% \mathrm{HM}, 0.20 \% \mathrm{SM}$ and $0.20 \% \mathrm{HM}$ has no effect on PER of whole trial (0 - 6 week) and starter phase (0 - 3 week), but during finisher period (4 - 6 week), PER was highest $(\mathrm{P}<0.05)$ in $0.15 \%$ and $0.20 \%$ SM supplemented groups with no significant difference in HM supplemented groups. The information on this aspect is scanty, so the results cannot be discussed due to lack of literature. 
Table 1: Ingredients and chemical composition of experimental diet for broiler i.e., pre-starter, starter and finisher.

\begin{tabular}{|c|c|c|c|c|c|c|c|c|c|c|c|c|c|c|c|}
\hline \multirow{3}{*}{ Attributes } & \multicolumn{15}{|c|}{ Experimental diet } \\
\hline & \multicolumn{5}{|c|}{ Pre-starter } & \multicolumn{5}{|c|}{ Starter } & \multicolumn{5}{|c|}{ Finisher } \\
\hline & $\mathrm{C}$ & $\mathrm{T}_{1}$ & $\mathrm{~T}_{2}$ & $\mathrm{~T}_{3}$ & $\mathrm{~T}_{4}$ & $\mathrm{C}$ & $\mathrm{T}_{1}$ & $\mathrm{~T}_{2}$ & $\mathrm{~T}_{3}$ & $\mathrm{~T}_{4}$ & $\mathrm{C}$ & $\mathrm{T}_{1}$ & $\mathrm{~T}_{2}$ & $\mathrm{~T}_{3}$ & $\mathrm{~T}_{4}$ \\
\hline \multicolumn{16}{|c|}{ Ingredient composition (\%) } \\
\hline Maize & 60.50 & 60.50 & 60.50 & 60.50 & 60.50 & 60.88 & 60.92 & 60.92 & 60.92 & 60.92 & 67.50 & 67.51 & 67.51 & 67.51 & 67.51 \\
\hline Meat bone meal & 5.00 & 5.00 & 5.00 & 5.00 & 5.00 & 5.00 & 5.00 & 5.00 & 5.00 & 5.00 & 5.00 & 5.00 & 5.00 & 5.00 & 5.00 \\
\hline Soya bean meal & 31.08 & 31.11 & 31.11 & 31.11 & 31.11 & 30.36 & 30.36 & 30.36 & 30.36 & 30.36 & 24.50 & 24.50 & 24.50 & 24.50 & 24.50 \\
\hline Salt & 0.25 & 0.25 & 0.25 & 0.25 & 0.25 & 0.25 & 0.25 & 0.25 & 0.25 & 0.25 & 0.25 & 0.25 & 0.25 & 0.25 & 0.25 \\
\hline Sodium bicarbonate & 0.01 & 0.01 & 0.01 & 0.01 & 0.01 & 0.01 & 0.01 & 0.01 & 0.01 & 0.01 & 0.01 & 0.01 & 0.01 & 0.01 & 0.01 \\
\hline Soya bean oil & 2.00 & 2.00 & 2.00 & 2.00 & 2.00 & 2.60 & 2.60 & 2.60 & 2.60 & 2.60 & 2.20 & 2.20 & 2.20 & 2.20 & 2.20 \\
\hline DL-Methionine & 0.17 & 0.12 & 0.08 & 0.04 & 0.00 & 0.13 & 0.09 & 0.06 & 0.03 & 0.00 & 0.09 & 0.07 & 0.04 & 0.02 & 0.00 \\
\hline Herbal methionine & 0.00 & 0.04 & 0.08 & 0.12 & 0.16 & 0.00 & 0.03 & 0.06 & 0.09 & 0.12 & 0.00 & 0.02 & 0.05 & 0.07 & 0.09 \\
\hline Lysine & 0.12 & 0.12 & 0.12 & 0.12 & 0.12 & 0.00 & 0.00 & 0.00 & 0.00 & 0.00 & 0.00 & 0.00 & 0.00 & 0.00 & 0.00 \\
\hline DCP & 0.13 & 0.13 & 0.13 & 0.13 & 0.13 & 0.00 & 0.00 & 0.00 & 0.00 & 0.00 & 0.00 & 0.00 & 0.00 & 0.00 & 0.00 \\
\hline LSP & 0.59 & 0.59 & 0.59 & 0.59 & 0.59 & 0.54 & 0.54 & 0.54 & 0.54 & 0.54 & 0.29 & 0.29 & 0.29 & 0.29 & 0.29 \\
\hline Vitamin supplement & 0.05 & 0.05 & 0.05 & 0.05 & 0.05 & 0.05 & 0.05 & 0.05 & 0.05 & 0.05 & 0.05 & 0.05 & 0.05 & 0.05 & 0.05 \\
\hline Trace minerals & 0.10 & 0.10 & 0.10 & 0.10 & 0.10 & 0.10 & 0.10 & 0.10 & 0.10 & 0.10 & 0.10 & 0.10 & 0.10 & 0.10 & 0.10 \\
\hline \multicolumn{16}{|c|}{ Chemical composition (\% DM) } \\
\hline $\mathrm{OM}$ & 94.86 & 94.47 & 94.29 & 94.02 & 93.78 & 95.37 & 95.29 & 95.14 & 95.11 & 94.87 & 96.50 & 94.89 & 95.76 & 94.08 & 94.82 \\
\hline $\mathrm{CP}$ & 21.94 & 21.87 & 21.76 & 21.62 & 21.89 & 21.67 & 21.45 & 21.39 & 21.28 & 21.24 & 19.86 & 19.41 & 19.34 & 19.22 & 19.17 \\
\hline $\mathrm{EE}$ & 3.29 & 3.26 & 3.34 & 3.41 & 3.56 & 4.23 & 4.32 & 4.37 & 4.48 & 4.59 & 5.01 & 5.22 & 5.18 & 5.24 & 5.27 \\
\hline $\mathrm{CF}$ & 4.08 & 4.14 & 4.05 & 4.18 & 4.22 & 4.20 & 4.26 & 4.29 & 4.31 & 4.19 & 4.24 & 4.40 & 4.35 & 4.38 & 4.44 \\
\hline TA & 5.14 & 5.53 & 5.71 & 5.98 & 6.22 & 4.63 & 4.71 & 4.86 & 4.89 & 5.13 & 3.50 & 5.11 & 4.24 & 5.92 & 5.18 \\
\hline NFE & 65.55 & 65.20 & 65.14 & 64.81 & 64.11 & 65.27 & 65.26 & 65.09 & 65.04 & 64.85 & 67.39 & 65.86 & 66.89 & 65.24 & 65.94 \\
\hline AIA & 1.47 & 1.40 & 1.49 & 1.48 & 1.96 & 1.46 & 1.48 & 1.43 & 1.94 & 1.41 & 1.49 & 1.93 & 1.47 & 1.92 & 1.31 \\
\hline $\mathrm{Ca}$ & 1.17 & 1.15 & 1.17 & 1.13 & 1.20 & 1.02 & 1.04 & 1.09 & 1.02 & 1.06 & 0.98 & 0.95 & 0.90 & 0.92 & 0.95 \\
\hline $\mathrm{P}$ & 0.85 & 0.84 & 0.82 & 0.87 & 0.85 & 0.80 & 0.78 & 0.74 & 0.76 & 0.80 & 0.76 & 0.73 & 0.75 & 0.74 & 0.78 \\
\hline ME (kcal $/ \mathrm{kg})$ calcu & 3000 & 3000 & 3000 & 3000 & 3000 & 3050 & 3050 & 3050 & 3050 & 3050 & 3100 & 3100 & 3100 & 3100 & 3100 \\
\hline
\end{tabular}


Table 2: Comparative efficacy of herbal methionine vis-a-vis synthetic methionine on cumulative feed intake ( $\mathrm{g}$ ), body weight gain ( $\mathrm{g}$ ), feed conversion ratio and protein efficiency ratio in broiler chicken

\begin{tabular}{|c|c|c|c|c|c|c|}
\hline \multirow{2}{*}{ Days } & \multicolumn{5}{|c|}{ Group } & \multirow{2}{*}{ Mean \pm SE } \\
\hline & $\mathrm{C}$ & $\mathrm{T}_{1}$ & $\mathrm{~T}_{2}$ & $\mathrm{~T}_{3}$ & $\mathrm{~T}_{4}$ & \\
\hline \multicolumn{7}{|c|}{ Feed intake (g) } \\
\hline $0-2$ week & $537.77 \pm 13.30$ & $540.47 \pm 21.74$ & $549.53 \pm 10.82$ & $542.90 \pm 16.81$ & $528.92 \pm 18.67$ & $539.92 \pm 6.82$ \\
\hline $2-3$ week & $642.19 \pm 23.44$ & $678.22 \pm 23.78$ & $658.55 \pm 16.76$ & $676.72 \pm 13.77$ & $657.17 \pm 9.45$ & $662.57 \pm 7.90$ \\
\hline $3-6$ week & $2723.22 \pm 33.64$ & $2683.03 \pm 33.65$ & $2649.19 \pm 26.40$ & $2726.99 \pm 63.06$ & $2753.26 \pm 55.88$ & $2707.14 \pm 19.71$ \\
\hline $0-6$ week & $3903.18 \pm 24.81$ & $3901.72 \pm 49.59$ & $3857.27 \pm 29.97$ & $3946.60 \pm 54.95$ & $3939.35 \pm 73.23$ & $3909.62 \pm 21.07$ \\
\hline \multicolumn{7}{|c|}{ Body weight gain (g) } \\
\hline $0-2$ week & $357.34^{b} \pm 8.46$ & $346.45^{\mathrm{ab}} \pm 7.88$ & $348.33^{\mathrm{ab}} \pm 4.00$ & $344.00^{\mathrm{ab}} \pm 8.90$ & $318.81^{\mathrm{a}} \pm 13.79$ & $342.98 \pm 4.69$ \\
\hline $2-3$ week & $358.01 \pm 23.62$ & $368.88 \pm 17.85$ & $350.81 \pm 23.01$ & $352.46 \pm 14.35$ & $314.18 \pm 10.30$ & $348.87 \pm 8.50$ \\
\hline $3-6$ week & $1324.25^{\mathrm{b}} \pm 39.09$ & $1203.87^{\mathrm{a}} \pm 19.59$ & $1163.79^{\mathrm{a}} \pm 11.72$ & $1181.67^{\mathrm{a}} \pm 32.85$ & $1161.78^{\mathrm{a}} \pm 28.77$ & $1207.07 \pm 17.82$ \\
\hline $0-6$ week & $2039.60^{\mathrm{c}} \pm 27.10$ & $1919.19^{\mathrm{b}} \pm 22.44$ & $1862.93^{\mathrm{ab}} \pm 35.76$ & $1878.12^{\mathrm{ab}} \pm 30.42$ & $1794.77^{\mathrm{a}} \pm 47.49$ & $1898.92 \pm 22.91$ \\
\hline \multicolumn{7}{|c|}{ Feed conversion ratio } \\
\hline $0-2$ week & $1.50^{\mathrm{a}} \pm 0.02$ & $1.56^{\mathrm{ab}} \pm 0.04$ & $1.58^{\mathrm{ab}} \pm 0.04$ & $1.58^{\mathrm{ab}} \pm 0.01$ & $1.67^{\mathrm{b}} \pm 0.09$ & $1.58 \pm 0.02$ \\
\hline $2-3$ week & $1.89^{\mathrm{a}} \pm 0.05$ & $1.84^{\mathrm{a}} \pm 0.03$ & $1.90^{\mathrm{ab}} \pm 0.13$ & $1.93^{\mathrm{ab}} \pm 0.04$ & $2.10^{\mathrm{b}} \pm 0.04$ & $1.91 \pm 0.04$ \\
\hline $3-6$ week & $2.06^{a} \pm 0.04$ & $2.23^{\mathrm{b}} \pm 0.19$ & $2.28^{\mathrm{b}} \pm 0.03$ & $2.31^{b c} \pm 0.03$ & $2.37^{c} \pm 0.02$ & $2.25 \pm 0.03$ \\
\hline $0-6$ week & $1.91^{\mathrm{a}} \pm 0.02$ & $2.03^{\mathrm{b}} \pm 0.01$ & $2.07^{\mathrm{b}} \pm 0.04$ & $2.10^{\mathrm{b}} \pm 0.01$ & $2.20^{\mathrm{c}} \pm 0.04$ & $2.06 \pm 0.02$ \\
\hline \multicolumn{7}{|c|}{ Protein efficiency ratio } \\
\hline $0-2$ week & $3.02^{\mathrm{b}} \pm 0.04$ & $2.92^{\mathrm{ab}} \pm 0.09$ & $2.89^{\mathrm{ab}} \pm 0.08$ & $2.88^{\mathrm{ab}} \pm 0.02$ & $2.75^{\mathrm{a}} \pm 0.13$ & $2.89 \pm 0.04$ \\
\hline $2-3$ week & $2.59^{b} \pm 0.08$ & $2.53^{\mathrm{ab}} \pm 0.03$ & $2.48^{\mathrm{ab}} \pm 0.18$ & $2.41^{\mathrm{ab}} \pm 0.05$ & $2.22^{\mathrm{a}} \pm 0.04$ & $2.44 \pm 0.05$ \\
\hline $3-6$ week & $2.49^{c} \pm 0.05$ & $2.30^{\mathrm{b}} \pm 0.02$ & $2.25^{\mathrm{ab}} \pm 0.02$ & $2.22^{\mathrm{ab}} \pm 0.02$ & $2.16^{\mathrm{a}} \pm 0.02$ & $2.29 \pm 0.03$ \\
\hline $0-6$ week & $2.59^{\mathrm{c}} \pm 0.02$ & $2.44^{\mathrm{b}} \pm 0.01$ & $2.39^{\mathrm{b}} \pm 0.05$ & $2.36^{\mathrm{b}} \pm 0.01$ & $2.26^{\mathrm{a}} \pm 0.04$ & $2.41 \pm 0.03$ \\
\hline
\end{tabular}

${ }^{\mathrm{abc}}$ Mean with different superscript within a row differ significantly $(\mathrm{P}<0.05)$

Table 3: Comparative efficacy of herbal methionine vis-a-vis synthetic methionine on carcass characteristics in broiler chicken

\begin{tabular}{|c|c|c|c|c|c|}
\hline \multirow{2}{*}{ Attributes } & \multicolumn{5}{|c|}{ Groups } \\
\hline & $\mathrm{C}$ & $\mathrm{T}_{1}$ & $\mathrm{~T}_{2}$ & $\mathrm{~T}_{3}$ & $\mathrm{~T}_{4}$ \\
\hline Live weight $(\mathrm{g})$ & $2084.38^{b} \pm 35.31$ & $1963.13^{\mathrm{ab}} \pm 19.50$ & $1853.75^{\mathrm{a}} \pm 67.40$ & $1981.88^{\mathrm{ab}} \pm 27.12$ & $1858.75^{\mathrm{a}} \pm 55.00$ \\
\hline Bled weight $(\mathrm{g})$ & $1962.50^{\mathrm{b}} \pm 35.08$ & $1861.88^{\mathrm{ab}} \pm 17.95$ & $1778.75^{\mathrm{a}} \pm 63.32$ & $1900.00^{\mathrm{ab}} \pm 29.43$ & $1773.75^{\mathrm{a}} \pm 51.07$ \\
\hline$\%$ of live weight & $94.15^{\mathrm{a}} \pm 0.30$ & $94.85^{\mathrm{ab}} \pm 0.14$ & $95.98^{\mathrm{c}} \pm 0.23$ & $95.85^{\mathrm{c}} \pm 0.25$ & $95.44^{\mathrm{bc}} \pm 0.26$ \\
\hline De-feathered weight without skin (g) & $1601.00^{\mathrm{b}} \pm 18.52$ & $1521.25^{\mathrm{ab}} \pm 13.02$ & $1423.75^{\mathrm{a}} \pm 54.02$ & $1523.50^{\mathrm{ab}} \pm 16.07$ & $1432.50^{\mathrm{a}} \pm 53.34$ \\
\hline$\%$ of live weight & $76.87 \pm 0.67$ & $77.50 \pm 0.28$ & $76.77 \pm 0.38$ & $76.91 \pm 0.52$ & $76.95 \pm 0.68$ \\
\hline Feathers weight with skin (g) & $361.50 \pm 21.70$ & $340.63 \pm 8.63$ & $355.00 \pm 12.32$ & $376.50 \pm 16.52$ & $341.25 \pm 5.49$ \\
\hline$\%$ of live weight & $17.28^{\mathrm{a}} \pm 0.08$ & $17.34^{\mathrm{ab}} \pm 0.35$ & $19.20^{b} \pm 0.47$ & $18.95^{\mathrm{ab}} \pm 0.60$ & $18.49^{\mathrm{ab}} \pm 0.68$ \\
\hline GIT $(\mathrm{g})$ & $132.31 \pm 10.16$ & $120.06 \pm 5.17$ & $113.21 \pm 8.12$ & $122.91 \pm 6.40$ & $117.65 \pm 11.17$ \\
\hline$\%$ of live weight & $6.31 \pm 0.38$ & $6.11 \pm 0.22$ & $6.08 \pm 0.26$ & $6.18 \pm 0.25$ & $6.26 \pm 0.40$ \\
\hline Gizzard weight (g) & $44.78 \pm 2.54$ & $41.36 \pm 1.60$ & $40.34 \pm 3.16$ & $42.93 \pm 2.85$ & $41.01 \pm 3.00$ \\
\hline$\%$ of live weight & $2.14 \pm 0.09$ & $2.11 \pm 0.07$ & $2.15 \pm 0.09$ & $2.16 \pm 0.13$ & $2.19 \pm 0.10$ \\
\hline Dressing \% & $66.03 \pm 0.96$ & $66.86 \pm 0.37$ & $65.79 \pm 0.52$ & $66.07 \pm 0.75$ & $65.67 \pm 0.47$ \\
\hline
\end{tabular}

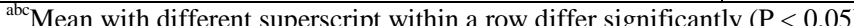


Figure 1: Feather scoring system applied in broilers

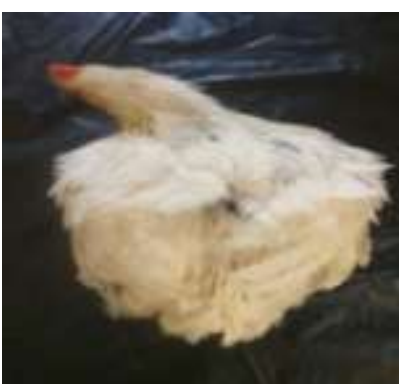

C:- SCORE 1

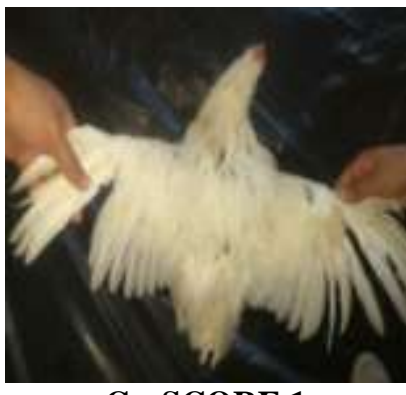

C:- SCORE 1

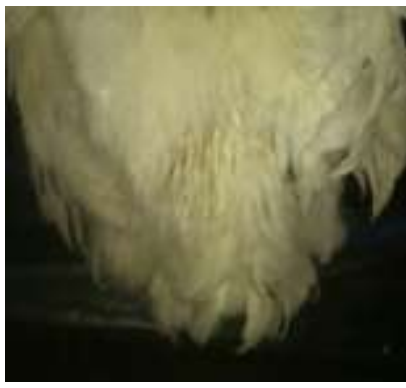

C:- SCORE 1

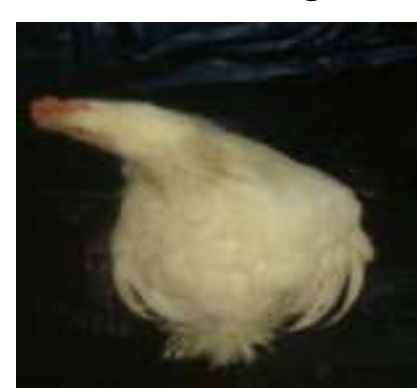

$\mathrm{T}_{1}$ : SCORE 0

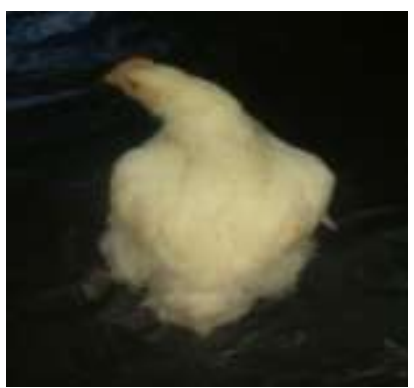

$T_{2}$ : SCORE 0

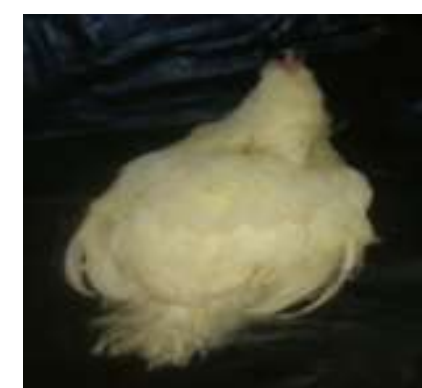

$T_{3}$ : SCORE 0

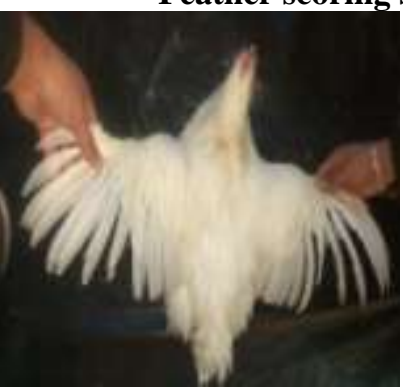

$\mathrm{T}_{1}$ : SCORE 0

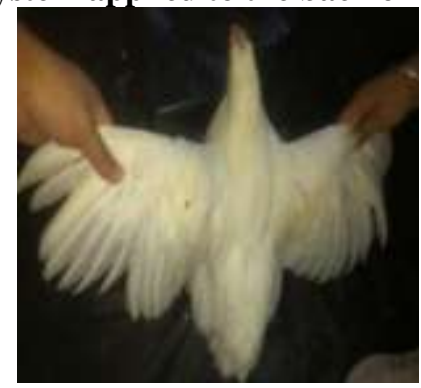

$T_{2}$ : SCORE 0

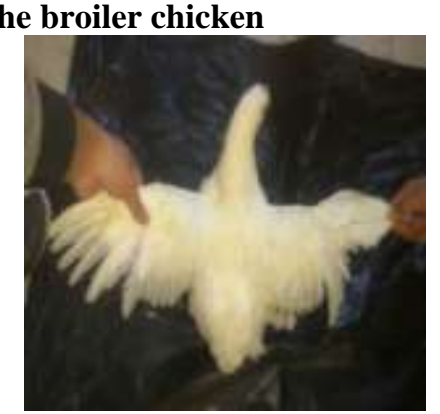

$T_{3}$ : SCORE 0

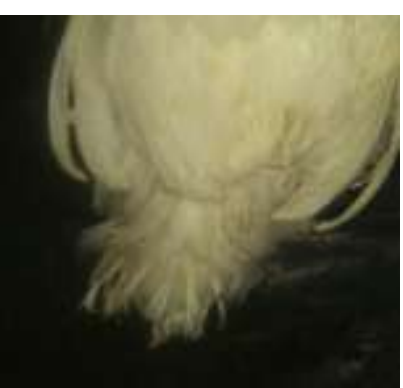

$\mathrm{T}_{1}$ : SCORE 0

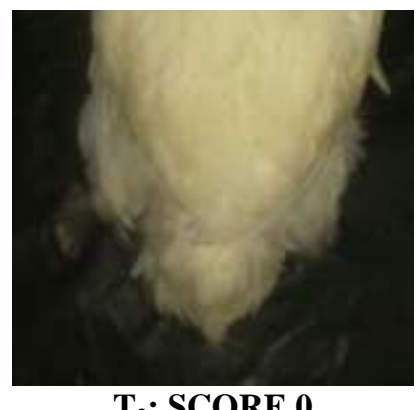

$T_{2}:$ SCORE 0

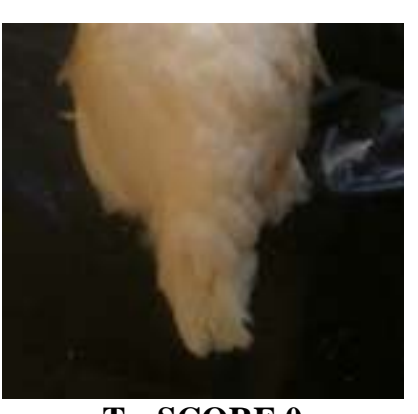

$\mathrm{T}_{3}$ : SCORE 0

er chicken

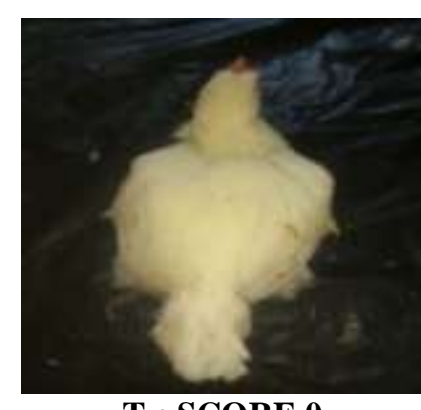

$\mathrm{T}_{4}$ : SCORE 0

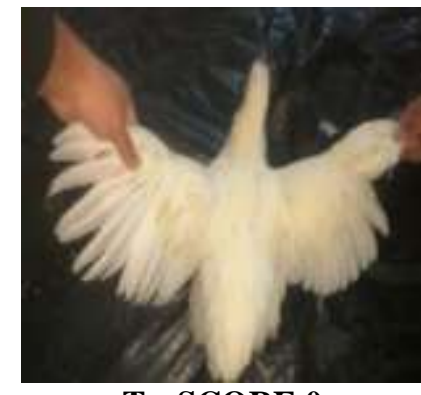

$T_{4}$ : SCORE 0

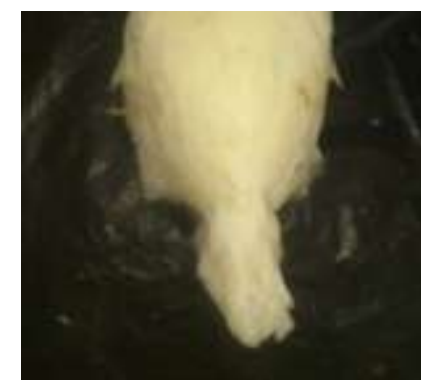

$\mathrm{T}_{4}$ : SCORE 0 
Table 4: Comparative efficacy of herbal methionine vis-a-vis synthetic methionine on weight of cut up parts of broiler chicken

\begin{tabular}{|l|l|l|l|l|l|}
\hline \multirow{2}{*}{ Attributes } & \multicolumn{5}{|c|}{ Groups } \\
\cline { 2 - 6 } & $\mathrm{C}$ & $\mathrm{T}_{1}$ & $\mathrm{~T}_{2}$ & $\mathrm{~T}_{3}$ & $\mathrm{~T}_{4}$ \\
\hline Head weight $(\mathrm{g})$ & $66.44^{\mathrm{b}} \pm 1.41$ & $59.03^{\mathrm{a}} \pm 0.80$ & $58.08^{\mathrm{a}} \pm 2.19$ & $59.42^{\mathrm{a}} \pm 0.63$ & $58.56^{\mathrm{a}} \pm 1.51$ \\
\hline \% of live weight & $3.19 \pm 0.07$ & $3.01 \pm 0.05$ & $3.15 \pm 0.10$ & $3.00 \pm 0.05$ & $3.16 \pm 0.06$ \\
\hline Shank weight $(\mathrm{g})$ & $72.66 \pm 2.15$ & $68.32 \pm 1.45$ & $71.12 \pm 2.84$ & $71.62 \pm 2.25$ & $72.99 \pm 2.32$ \\
\hline \% of live weight & $3.49^{\mathrm{a}} \pm 0.08$ & $3.49^{\mathrm{a}} \pm 0.09$ & $3.86^{\mathrm{b}} \pm 0.17$ & $3.62^{\mathrm{ab}} \pm 0.12$ & $3.93^{\mathrm{b}} \pm 0.11$ \\
\hline Neck weight $(\mathrm{g})$ & $56.74 \pm 2.74$ & $55.59 \pm 3.32$ & $51.07 \pm 3.07$ & $57.96 \pm 3.51$ & $59.48 \pm 3.70$ \\
\hline \% of live weight & $2.72^{\mathrm{a}} \pm 0.11$ & $2.83^{\mathrm{ab}} \pm 0.16$ & $2.74^{\mathrm{a}} \pm 0.08$ & $2.93^{\mathrm{ab}} \pm 0.18$ & $3.18^{\mathrm{b}} \pm 0.12$ \\
\hline Legs weight $(\mathrm{g})$ & $202.49^{\mathrm{b}} \pm 6.03$ & $191.32^{\mathrm{ab}} \pm 6.21$ & $177.32^{\mathrm{a}} \pm 9.61$ & $184.82^{\mathrm{ab}} \pm 3.59$ & $171.98^{\mathrm{a}} \pm 5.30$ \\
\hline \% of live weight & $9.70 \pm 0.15$ & $9.73 \pm 0.25$ & $9.54 \pm 0.22$ & $9.32 \pm 0.10$ & $9.26 \pm 0.14$ \\
\hline Thigh weight $(\mathrm{g})$ & $196.13^{\mathrm{b}} \pm 7.57$ & $188.61^{\mathrm{ab}} \pm 4.04$ & $170.67^{\mathrm{a}} \pm 6.48$ & $185.49^{\mathrm{ab}} \pm 9.63$ & $179.61^{\mathrm{ab}} \pm 10.44$ \\
\hline \% of live weight & $9.40 \pm 0.28$ & $9.61 \pm 0.18$ & $9.21 \pm 0.15$ & $9.33 \pm 0.39$ & $9.61 \pm 0.28$ \\
\hline Breast weight $(\mathrm{g})$ & $479.30^{\mathrm{c}} \pm 13.9$ & $428.89^{\mathrm{b}} \pm 10.16$ & $383.55^{\mathrm{a}} \pm 17.99$ & $398.89^{\mathrm{ab}} \pm 12.19$ & $383.27^{\mathrm{a}} \pm 11.80$ \\
\hline \% of live weight & $23.03^{\mathrm{c}} \pm 0.72$ & $21.84^{\mathrm{bc}} \pm 0.43$ & $20.65^{\mathrm{ab}} \pm 0.51$ & $20.11^{\mathrm{a}} \pm 0.44$ & $20.66^{\mathrm{ab}} \pm 0.51$ \\
\hline Wings weight $(\mathrm{g})$ & $145.66 \pm 4.95$ & $145.52 \pm 3.09$ & $141.27 \pm 9.03$ & $145.50 \pm 3.32$ & $145.30 \pm 3.21$ \\
\hline \% of live weight & $6.99^{\mathrm{a}} \pm 0.23$ & $7.42^{\mathrm{ab}} \pm 0.16$ & $7.60^{\mathrm{ab}} \pm 0.32$ & $7.34^{\mathrm{ab}} \pm 0.14$ & $7.84^{\mathrm{b}} \pm 0.17$ \\
\hline
\end{tabular}

${ }^{\text {abc } M e a n ~ w i t h ~ d i f f e r e n t ~ s u p e r s c r i p t ~ w i t h i n ~ a ~ r o w ~ d i f f e r ~ s i g n i f i c a n t l y ~}(\mathrm{P}<0.05)$

Table 5: Comparative efficacy of herbal methionine vis-a-vis synthetic methionine on weight of immunological and other visceral organs of broiler chicken

\begin{tabular}{|c|c|c|c|c|c|}
\hline \multirow[t]{2}{*}{ Attributes } & \multicolumn{5}{|l|}{ Group } \\
\hline & $\mathrm{C}$ & $\mathrm{T}_{1}$ & $\mathrm{~T}_{2}$ & $\mathrm{~T}_{3}$ & $\mathrm{~T}_{4}$ \\
\hline \multicolumn{6}{|c|}{ Immunological organs } \\
\hline Bursa weight $(\mathrm{g})$ & $6.82 \pm 0.59$ & $6.06 \pm 0.61$ & $5.58 \pm 0.31$ & $6.11 \pm 0.41$ & $5.75 \pm 0.46$ \\
\hline$\%$ of live weight & $0.33 \pm 0.02$ & $0.31 \pm 0.03$ & $0.30 \pm 0.01$ & $0.31 \pm 0.02$ & $0.31 \pm 0.02$ \\
\hline Spleen weight $(\mathrm{g})$ & $2.40^{\mathrm{a}} \pm 0.09$ & $2.86^{\mathrm{ab}} \pm 0.15$ & $3.04^{\mathrm{ab}} \pm 0.20$ & $3.12^{\mathrm{ab}} \pm 0.38$ & $3.58^{b} \pm 0.28$ \\
\hline$\%$ of live weight & $0.12^{\mathrm{a}} \pm 0.01$ & $0.15^{\mathrm{ab}} \pm 0.01$ & $0.17^{\mathrm{bc}} \pm 0.01$ & $0.16^{\mathrm{bc}} \pm 0.02$ & $0.19^{c} \pm 0.02$ \\
\hline Liver weight (g) & $46.71 \pm 2.99$ & $42.51 \pm 1.40$ & $40.31 \pm 1.57$ & $45.17 \pm 1.90$ & $42.70 \pm 2.95$ \\
\hline$\%$ of live weight & $2.23 \pm 0.10$ & $2.16 \pm 0.06$ & $2.19 \pm 0.11$ & $2.28 \pm 0.09$ & $2.28 \pm 0.10$ \\
\hline \multicolumn{6}{|l|}{ Visceral organs } \\
\hline Heart weight $(\mathrm{g})$ & $11.65 \pm 0.77$ & $10.81 \pm 0.83$ & $10.29 \pm 0.70$ & $10.17 \pm 0.62$ & $10.01 \pm 0.56$ \\
\hline$\%$ of live weight & $0.56 \pm 0.03$ & $0.55 \pm 0.04$ & $0.55 \pm 0.02$ & $0.51 \pm 0.03$ & $0.54 \pm 0.01$ \\
\hline Lungs weight (g) & $12.62 \pm 0.54$ & $11.68 \pm 0.49$ & $10.98 \pm 0.85$ & $11.29 \pm 0.78$ & $11.03 \pm 0.90$ \\
\hline$\%$ of live weight & $0.61 \pm 0.03$ & $0.59 \pm 0.02$ & $0.58 \pm 0.03$ & $0.57 \pm 0.03$ & $0.59 \pm 0.04$ \\
\hline Kidney weight (g) & $9.02 \pm 0.68$ & $9.51 \pm 0.55$ & $9.47 \pm 0.88$ & $9.90 \pm 0.59$ & $9.57 \pm 0.62$ \\
\hline$\%$ of live weight & $0.43 \pm 0.03$ & $0.49 \pm 0.03$ & $0.51 \pm 0.03$ & $0.50 \pm 0.03$ & $0.51 \pm 0.02$ \\
\hline
\end{tabular}

Table 6: Comparative efficacy of herbal methionine vis-a-vis synthetic methionine on length of internal organs (cm) in broiler chicken

\begin{tabular}{|l|c|c|c|c|c|c|}
\hline \multirow{2}{*}{ Parameters } & \multicolumn{5}{|c|}{ Group } & \multirow{2}{*}{ Mean $\pm \mathrm{SE}$} \\
\cline { 2 - 7 } & $\mathrm{C}$ & $\mathrm{T}_{1}$ & $\mathrm{~T}_{2}$ & $\mathrm{~T}_{3}$ & $\mathrm{~T}_{4}$ & \\
\hline Duodenum $(\mathrm{cm})$ & $31.38 \pm 1.40$ & $30.13 \pm 1.47$ & $30.06 \pm 1.46$ & $30.13 \pm 1.46$ & $30.25 \pm 1.58$ & $30.39 \pm 0.63$ \\
\hline Jejunum $(\mathrm{cm})$ & $50.06 \pm 3.15$ & $53.75 \pm 4.35$ & $51.75 \pm 2.07$ & $50.13 \pm 1.93$ & $51.00 \pm 2.10$ & $51.34 \pm 1.24$ \\
\hline Ileum $(\mathrm{cm})$ & $80.38 \pm 3.06$ & $81.88 \pm 2.95$ & $80.13 \pm 5.26$ & $79.38 \pm 3.23$ & $84.13 \pm 3.63$ & $81.18 \pm 1.60$ \\
\hline Caecum $(\mathrm{cm})$ & $16.88 \pm 0.40$ & $16.75 \pm 0.77$ & $16.38 \pm 0.77$ & $17.63 \pm 0.46$ & $17.69 \pm 0.70$ & $17.06 \pm 0.28$ \\
\hline
\end{tabular}

Table 7: Comparative efficacy of herbal methionine vis-a-vis synthetic methionine on Feather quality (with skin) in broiler chicken

\begin{tabular}{|l|l|l|l|l|l|}
\hline \multirow{2}{*}{ Particulars } & \multicolumn{5}{|c|}{ Group } \\
\cline { 2 - 6 } & $\mathrm{C}$ & $\mathrm{T}_{1}$ & $\mathrm{~T}_{2}$ & $\mathrm{~T}_{3}$ & $\mathrm{~T}_{4}$ \\
\hline Feather colour & Whitish with black spot & White & White & White & White \\
\hline Feather weight (\% of live weight) & $5.26^{\mathrm{a}} \pm 0.05$ & $5.35^{\mathrm{ab}} \pm 0.04$ & $5.48^{\mathrm{b}} \pm 0.03$ & $5.63^{\mathrm{c}} \pm 0.05$ & $5.81^{\mathrm{d}} \pm 0.06$ \\
\hline \multicolumn{7}{|c|}{ Feather scoring } & $0.25^{\mathrm{a}} \pm 0.16$ & $0.13^{\mathrm{a}} \pm 0.13$ & $0.13^{\mathrm{a}} \pm 0.13$ & $0.00^{\mathrm{a}} \pm 0.00$ \\
\hline Back & $0.75^{\mathrm{b}} \pm 0.16$ & $0.38^{\mathrm{a}} \pm 0.18$ & $0.25^{\mathrm{a}} \pm 0.16$ & $0.38^{\mathrm{a}} \pm 0.18$ & $0.13^{\mathrm{a}} \pm 0.13$ \\
\hline Wing & $0.88^{\mathrm{b}} \pm 0.13$ & $0.75^{\mathrm{b}} \pm 0.16$ & $0.13^{\mathrm{a}} \pm 0.13$ & $0.00^{\mathrm{a}} \pm 0.00$ & $0.00^{\mathrm{a}} \pm 0.00$ \\
\hline Tail & $1.00^{\mathrm{b}} \pm 0.00$ &
\end{tabular}


Table 8: Comparative efficacy of herbal methionine vis-a-vis synthetic methionine on cost of unit weight gain among dietary treatments

\begin{tabular}{|c|c|c|c|c|c|c|}
\hline \multirow{2}{*}{ Attributes } & \multicolumn{5}{|c|}{ Treatment } & \multirow{2}{*}{ Mean } \\
\hline & $\mathrm{C}$ & $\mathrm{T}_{1}$ & $\mathrm{~T}_{2}$ & $\mathrm{~T}_{3}$ & $\mathrm{~T}_{4}$ & \\
\hline Prestarter feed cost (Rs/kg) & 27.70 & 27.52 & 27.41 & 27.29 & 27.20 & $27.42 \pm 0.04$ \\
\hline Starter feed cost $(\mathrm{Rs} / \mathrm{kg})$ & 27.78 & 27.63 & 27.55 & 27.46 & 27.38 & $27.56 \pm 0.03$ \\
\hline Finisher feed cost $(\mathrm{Rs} / \mathrm{kg})$ & 25.82 & 25.72 & 25.65 & 25.59 & 25.55 & $25.67 \pm 0.02$ \\
\hline $0-2$ wk feed cost gain & $24.00 \pm 0.32$ & $23.36 \pm 0.69$ & $23.16 \pm 0.65$ & $23.23 \pm 0.18$ & $22.22 \pm 1.07$ & $23.19 \pm 0.29$ \\
\hline $2-3$ wk feed cost gain & $20.01^{b} \pm 0.60$ & $19.66^{\mathrm{ab}} \pm 0.27$ & $19.38^{\mathrm{ab}} \pm 1.43$ & $18.95^{\mathrm{ab}} \pm 0.42$ & $17.45^{\mathrm{a}} \pm 0.35$ & $19.09 \pm 0.36$ \\
\hline $3-6$ wk feed cost gain & $18.82^{\mathrm{c}} \pm 0.35$ & $17.45^{\mathrm{b}} \pm 0.15$ & $17.13^{\mathrm{ab}} \pm 0.19$ & $16.93^{\mathrm{ab}} \pm 0.22$ & $16.51^{\mathrm{a}} \pm 0.17$ & $17.37 \pm 0.20$ \\
\hline $0-6$ wk feed cost gain & $32.47 \pm 0.48$ & $31.76 \pm 0.62$ & $31.27 \pm 0.48$ & $31.77 \pm 0.34$ & $31.97 \pm 0.69$ & $31.85 \pm 0.23$ \\
\hline
\end{tabular}

Data on carcass characteristics and organ weights are presented in Table 3. In the present study carcass yields viz. live weight and bled weight of broiler birds of different dietary treatments was significantly higher in $\mathrm{C}$ than $\mathrm{T}_{2}$ and $\mathrm{T}_{4}$ with intermediate values for $\mathrm{T}_{1}$ and $\mathrm{T}_{3}$. But bled weight (\% of live weight) was higher in $\mathrm{HM}$ fed groups. Defeathered weight of birds without skin (g) was higher in SM supplemented group with variable values in rest of the groups. The weight of GIT ( $\mathrm{g}, \%$ live weight), gizzard (g, \% live weight) and dressing percentage were not influenced by different dietary treatments. These findings are in agreement with the reports of Kumar et al. (2014), who also stated no effect of replacing SM with $50 \%$ HM on different carcass parameters viz. slaughter weight, carcass weight, carcass yield. Ahmed and Abbas (2015) and Makinde et al. (2017) also found no effect of replacing SM with HM on dressing percentage in broilers. However, Chattopadhyay et al. (2006) reported higher dressing percentage in birds fed diet supplemented with $15 \mathrm{~g} / \mathrm{kg} \mathrm{HM}$ fed group in comparison to $10 \mathrm{~g} / \mathrm{kg} \mathrm{SM}$ and $10 \mathrm{~g} / \mathrm{kg} \mathrm{HM}$ fed groups.

The weight of cut up parts viz. head, shank, neck, legs, thigh, breast, wings (g) were found higher in SM supplemented group in comparison to rest of the groups (Table 4). The higher body weight of birds of SM fed group might be the probable reason for heavier cut up parts. But weight of cut up parts when expressed as $\%$ of live weight; shank, neck and wing weight was found higher $(\mathrm{P}<0.05)$ in $100 \% \mathrm{HM}$ supplemented group. Similar conclusions were observed by earlier researchers (Kumari et al., 2012 and Makinde et al. 2017). As neck weight is determined separately in the present study, so no comparison can be done in this aspect. However, these results are contradictory to the findings of Chattopadhyay et al. (2006), who reported significantly (P < 0.05 ) higher breast yield $\%$ and thigh yield \% in birds fed diet supplemented with $15 \mathrm{~g} / \mathrm{kg} \mathrm{HM}$ fed group in comparison to $10 \mathrm{~g} / \mathrm{kg} \mathrm{SM}$ and $10 \mathrm{~g} / \mathrm{kg} \mathrm{HM}$ fed groups. According to Freitas et al. (2006), when dietary nutritional levels are properly evaluated, it is unlikely that carcass yield is influenced by the inclusion of alternative feedstuffs in experimental diets in which most of the nutritional parameters are similar in feed formulation. On the other hand, if the metabolizable energy content of a feedstuff and particularly net energy, are underestimated or overestimated, its inclusion in the diet may change energy protein ratio, consequently changing carcass and cuts composition. The similar nutritional contents of different diets are the probable reason for similar carcass yield.

The weights (in gram and $\%$ of live weight) of immunological organs like bursa and liver was found to be statistically similar, but spleen weight was significantly higher $(\mathrm{P}<0.05)$ in $\mathrm{T}_{4}$ than that of $\mathrm{C}$, whereas $\mathrm{T}_{1}, \mathrm{~T}_{2}$ and $T_{3}$ showed intermediate value of spleen weight (Table 5). Yuan et al. (2012) also found no effect of different sources and levels of methionine on immune organs (liver, spleen, bursa) when expressed as percent of body weight. These results equally agree with the findings of Ahmed and Abbas (2015). But Kumar et al. (2014) found higher $(\mathrm{P}<0.05)$ spleen and bursa yield on replacing SM with $50 \% \mathrm{HM}$.

Similarly visceral organs weights (Heart, lung, kidneys) did not differ significantly $(\mathrm{P}>0.05)$ irrespective of the groups (Table 5). Ahmed and Abbas (2015) and Mankinde et al. (2017) also found similar heart weight on replacing SM by graded levels of HM. But, Kumari et al. (2012) reported higher values of liver weight (\% live weight) in broilers fed different diets supplemented with either SM @ $2 \mathrm{~kg} / \mathrm{ton}$ feed or $1 \mathrm{~kg} / \mathrm{ton}$ $\mathrm{SM}$ and $1 \mathrm{~kg} /$ ton SM.

The length $(\mathrm{cm})$ of various parts of intestine like duodenum, jejunum, ileum and caecum did not differ significantly irrespective of the groups (Table 6), signifying no adverse effect of replacement of DL-methionine with graded levels of herbal methionine. Ahmed and Abbas (2015) stated lower $(\mathrm{P}<0.05)$ intestine length in $0.15 \% \mathrm{HM}$ fed group, but found no difference in intestine length of $0.15 \% \mathrm{SM}, 0.20 \% \mathrm{SM}, 0.20 \% \mathrm{HM}$ fed groups. Yuan et al. (2012) also found no effect of different sources and levels of methionine on weight of duodenum, jejunum plus ileum and caecum when expressed as percent of body weight.

Effect of replacement of herbal methionine vis-à-vis synthetic methionine on feather quality of broiler chicken is presented in Table 7. The results revealed that feathers of some control birds had black spots where as in rest of treatment groups feathers were whitish in color. The feather weight without skin (\% of live weight) was higher $(\mathrm{P}<0.05)$ in $\mathrm{T}_{4}(5.81 \%)$ followed by $\mathrm{T}_{3}(5.63 \%), \mathrm{T}_{2}(5.48 \%)$ and least in $\mathrm{C}(5.26 \%)$ but the feather weight of $\mathrm{T}_{1}(5.35 \%)$ was similar $(\mathrm{P}>0.05)$ to $\mathrm{C}$ and $\mathrm{T}_{2}$. The feather score of back and wings was 
significantly better $(\mathrm{P}<0.05)$ in herbal methionine supplemented groups (fully feathered; Figure 1$)$. But the feather score of tail region of broiler birds was 1.00 and 0.75 in $\mathrm{C}$ and $\mathrm{T}_{1}$ (rough) whereas in the rest of groups, tail region were fully feathered $\left(0.13,0.00\right.$ and 0.00 score in $\mathrm{T}_{2}, \mathrm{~T}_{3}$ and $\mathrm{T}_{4}$, respectively), indicating that herbal methionine improves the feather quality of broiler birds. Contrary to our findings, Halder and Roy (2007) found no difference in grade of feathers on supplementing broiler diets either with $1.2 \mathrm{~kg} / \mathrm{ton} \mathrm{SM}$ or $1.2 \mathrm{~kg} / \mathrm{ton} \mathrm{HM}$. The difference found in the two studies might be explained in the light of fact that in the current study different source of HM is used. The literature pertaining to feather quality is scanty, so the results cannot be discussed.

The cost $(\mathrm{Rs} / \mathrm{kg})$ of pre-starter feed and starter feed are comparable, but both these feeds are costlier than finisher feed (Table 8). This may be explainable to reason that crude protein in pre-starter $(21.59-21.94 \%)$ and starter feeds (21.24-21.67 \%) were higher than finisher feed (19.17-19.86\%). However, the cost of prestarter, starter and finisher feed had a decreasing trend by increasing levels of HM in feed. It was due to cheaper rates of HM (Rs. 150/kg) in comparison to SM (Rs. 500/kg).

Feed cost (Rs) per kg body weight gain during pre-starter phase ( 0 - 2 week) was statistically similar among different dietary treatments. Whereas during starter ( 2 - 3 week), feed cost was high in SM supplemented group than $100 \%$ HM supplemented group. Likewise in finisher phase (3 - 6 week), feed cost was low in $100 \%$ HM supplemented group than $25: 75$ and $100: 0$ SM : HM fed groups. SM is approximately three times costlier than HM which resulted in increased cost.

However, when economy was worked out based on overall period ( 0 - 6 weeks), the feed cost per $\mathrm{kg}$ body weight gain was statistically similar irrespective of different dietary treatments, but the values were numerically better in herbal methionine supplemented groups. Although HM is cheaper but lesser body weight is the probable reason for similar cost per $\mathrm{kg}$ body weight gain. Thus, based on results, it could be concluded that HM supplementation has no beneficial effect on economy. Makinde et al. (2017) also found no difference in cost of feed per kg weight but cost per kg feed reduced as concentration of HM increases. Kumari et al. (2012) too stated that economics of raising broiler chickens was better in birds fed diets supplemented with HM : SM @ 1 kg/ton each than birds fed diets supplemented with SM @ 2 kg/ton feed. Halder and Roy (2007) stated that replacement of SM by HM had significant effects over the economic returns (net return (Rs.), profit/bird, profit/kg gross weight). Contrary to our findings, Salome et al. (2010) observed that feed cost per kg weight gain was significantly $(\mathrm{P}<0.05)$ better for birds fed with SM than HM supplementation.

\section{Conclusion}

It may be concluded that HM supplementation has no beneficial effect on broiler performance except feather growth

\section{Acknowledgement}

Authors are thankful to Alphafacts heatlh solutions to provide METHIOMAX, free of cost.

\section{References}

1) Ahmed ME, Abbas TE, 2015. The effect of feeding herbal methionine versus DL-methionine supplemented diets on broiler performance and carcass characteristics. International Conference on Agricultural, Ecological and Medical Sciences. 2015: 23-27.

2) AOAC. Official Method of Analysis. $19^{\text {th }}$ edn. Association of official Analytical Chemist International, Washington, DC. 2012.

3) Bhutyal D, Khan N, Sharma RK, Mahajan V, 2019. Comparative efficacy of Herbal methionine vis-a-vis DLMethionine on nutrient digestibility and blood parameters of broiler chicken. International Journal of Life Sciences and Applied Science 1 (1): 19-28.

4) Binder M, 2003. Life cycle analysis of DL-methionine in broiler meat production. In: Information for the feed industry, Degussa feed additives, Hanau-Wolfgang, Germany, 1-8.

5) Chattopadhyay KM, Mondal K, Roy B, 2006. Comparative efficacy of DL-methionine and herbal methionine on performance of broiler chicken. International Journal of Poultry Science 5: 1034-1039.

6) Dafwang II, 2006. Meat, milk and eggs from farm wastes: Explorations in Animal Nutrition Research and Extension. An Inaugural lecture delivered by Dafwang, I.I. at Ahmadu Bello University, Zaria.

7) Dahiya JP, Hoehler D, Van Kessel AG, Drew MD, 2007. Effect of different dietary methionine sources on intestinal microbial populations in broiler chickens. Poultry Science 86 (11): 2358-66.

8) Duncan DB, 1995. New multiple range and ' $F$ '" test. Biometrics.11: 1-42.

9) Figge R, Soucaile P, Bestel-Corre G, 2010. Producing methionine without N-acetyl-methionine. Patent application publication, USA; No: US 2010/0047879 AI. 
10) Fikre A, Yami A, Kuo YH, Ahmed S, Gheysen G, Lambein F, 2010. Effect of methionine supplementation on physical responses and neurological symptoms in broiler chicks fed grass pea (Lathyrus sativus) based starter ration. Food and Chemical Toxicology 1: 11-17.

11) Freitas ER, Fuentes MFF, Santos Jr A, Guerreiro MEF, Espíndola GB, 2006. Farelo da castanha de caju em rações para frangos de corte. Pesquisa Agropecuária Brasileir 41 (6): 1001-1006.

12) Halder G, Roy B, 2007. Effect of herbal or synthetic methionine on performance cost benefit ratio, meat and feather quality of broiler chicken. International Journal of Poultry Science 12: 987-996.

13) ICAR, 2013. Nutrient Requirement of Animals-Poultry (ICAR-NIANP), Indian Council of Agricultural Research, New Delhi.

14) Kaur D, Nagra SS, Sodhi S, Dwivedi PN, 2013. Comparative performance of commercial broilers fed Herbomethione ${ }^{\circledR}$ as a replacement for DL-methionine in diet. Journal of Applied Animal Research 41 (4): 410-416.

15) Kretzschmar-McCluskey V, Fisher V, Tuijl OV, 2014. A practical guide to managing feather covers in broiler breeder females. Ross Tech Note 1-5.

16) Kumar VM, Ravikanth K, Miani S, 2014. Efficacy of supplementation of Methiorep, a herbal methionine supplement in improving growth, performance and carcass trait in broilers. World Journal of Pharmaceutical Research 3 (7): 716-725.

17) Kumari K, Tiwari SP, Subhankar N, Saxena MJ, Ravikanth K, Maini S, 2012. Studies on comparative efficacy of herbal amino acid (Methiorep) supplement with synthetic DL-methionine on broiler growth performance and carcass quality trait. International Journal of Scientific and Research Publications 2 (8): $1-6$.

18) Makinde OS, Ajibade AJ, Omotugba SK, Tumburawa MS, Ibe EA, Opoola E, Zaccheaus OS, 2017. Herbal methionine (Methiorep) improves growth performance of broiler chickens without affecting carcass characteristics and blood indices. Tropical and Subtropical Agroecosystem 20 (1): 131-139.

19) NRC, 1994. Nutrient Requirements of Poultry. $9^{\text {th }}$ Rev. Edn., National Academy Press, Washington DC.

20) Ojano-Dirain CP, Waldroup PW, 2002. Evaluation of lysine, methionine and threonine needs of broilers three to six week of age under moderate temperature stress. International Journal of Poultry Science 1: 16-21.

21) Sharma A, Ranjan S, 2015. Comparative effects of DL-Methionine and N-Methionine on growth performance and biochemical parameters of broilers. International Journal of Current Research 7 (7): 17689-17692.

22) Salome I, Dafwang, II, Bawa GS, 2010. Evaluation of methiorep as a substitute for methionine in broiler diets. International Journal of Poultry Science 9 (8): 809-812.

23) Snedecor GW, Cochran WG, 1994. Statistical Methods. $8^{\text {th }}$ Edition, The Iowa State University, Iowa, USA.

24) Yuan J, Karimi AJ, Goodgame SD, Lu C, Mussini FJ, Waldroup PW, 2012. Evaluation of herbal methionine source in broiler diets. International Journal of Poultry Science 11 (4): 247-250. 